\title{
Programas de postgrado en Ginecobstetricia: EI Residente toma la palabra
}

\author{
Alvaro Cano Quiñones M.D.*; Andrés Sarmiento Rodríguez M.D. **
}

\section{Introducción}

Pocas veces se le pide al residente la opinión acerca de su programa de especialización. Usualmente éste se califica de acuerdo con la óptica del docente, del hospital o de las directivas universitarias; sin tener en cuenta la opinión de la persona que directamente experimenta el contenido del programa. Es menos frecuente aun que se intente describir el perfil del residente de ginecobstetricia colombiano; sus deficiencias, fortalezas, vicios de formación y otras características que pueden afectar su desempeño. En que forma estas condiciones influyen en el profesional como producto final de este proceso de formación. A través de la siguiente encuesta pretendimos describir el perfil humano y académico del residente. Quisimos evaluar características nunca antes exploradas en forma objetiva y profunda, y como influyen en su rendimiento académico y asistencial. Especulamos que el resultado de esta encuesta deberá motivar a las Universidades y jefes de programas a revalorar el enfoque de los programas de residencia en beneficio del profesional y del ser humano.

\section{Material y métodos}

Se practicó un estudio descriptivo a nivel nacional con la colaboración de diferentes programas de residencia en Ginecología y Obstetricia. A partir de agosto de 1997 se enviaron por correo aéreo o se entregaron personalmente 160 encuestas a residentes de 14 programas radicados en Hospitales de Bogotá, Bucaramanga, Medellín, Cali, Popayán, Cartagena y Barranquilla. La aplicación de la encuesta a los residentes se hizo con la anuencia de los respectivos jefes de los programas. El formato inclúa 50 preguntas cerradas que exploraron cinco áreas específicas: (1) identificación, (2) características del programa de residencia, (3) perfil humano del residente, (4) aspectos económicos y (5) percepción de su papel dentro del contexto médico actual. El diseño de la encuesta permitía opcionalmente que conservara su carácter anónimo.

Residente IV, Gineco-obstetricia, Universidad El Bosque. Coordinador Académico U.M.M.F. Hospital Simón Bolívar. De la Unidad de Medicina Maternofetal, Servicio de Ginecología y Obstetricia, Hospital Simón Bolívar - Universidad El Bosque. Instituto de Diagnóstico Obstétrico y Ginecológico, Obgyn Lida. Santafé de Bogotá.
El instrumento evaluativo fue diseñado por un equipo multidisciplinario que incluyó a los autores con la asesoría directa de psicólogos de la Universidad El Bosque (Santafé de Bogotá) con experiencia en diseño de instrumentos evaluativos. Inicialmente la encuesta se validó en un grupo de lo residentes de nuestro programa. Los resultados iniciales y sus opiniones fueron tenidos en cuenta para perfeccionar el diseño del instrumento.

Los resultados fueron tabulados y analizados con el programa estadístico EPI INFO 6 (versión 6.04 B).

\section{Resultados}

En un lapso de seis meses se recuperaron un total de 115 encuestas correspondientes a 13 programas de residencia del país. Fue una constante en la mayoría de los programas encuestados, la demora para el diligenciamiento, recolección y devolución de los formularios. Este hecho se interpretó como parte de los resultados. Un solo programa de residencia no contestó la solicitud.

\section{Identificación y datos generales}

El promedio de edad de los encuestados fue de 29.2 años, con un rango de 20 a 39 años. El 77.5\% (88) fueron hombres, mientras el 22.5\% (27) fueron mujeres. El 35.5\% (39) correspondieron a casados. Con respecto al año de residencia, el $24.1 \%$ de los encuestados pertenecían al primer año, $45.5 \%$ al segundo año, $26.8 \%$ al tercer año y tan sólo $3.6 \%$, correspondían al cuarto año.

\section{Del programa de residencia}

El $37.4 \%$ de los residentes ingresaron al programa tras aplicar por primera vez. Un $11.5 \%$ requirieron 4 o más intentos. No existió diferencia estadística por sexos con relación al número de aplicaciones.

Previamente al ingreso al programa de residencia, el 52\% de los encuestados refirió no conocer su contenido o conocerlo en forma parcial y limitada. Unicamente el 11\% manifestaba conocerlo en forma completa.

Solamente un $20 \%$ de los residentes pertenecían a programas de 4 años. De este grupo, un 61\% manifestó no encontrar ninguna ventaja de esta duración en relación con el programa convencional de 3 años. Las razones más frecuentemente aducidas fueron: 
1. Un mismo contenido en un marco de tiempo mayor.

2. Carencia de un soporte docente.

3. Recargo de las labores asistenciales sin justificación académica.

En cuanto al contenido Académico del programa de residencia un 37.4\% lo calificó con 3 (en una escala de 1-5). Mientras un 10.4\% lo reprobó. Solamente el 15\% lo calificó con 5 (excelente). En relación con el cumplimiento de las expectativas académicas al ingreso del programa, el $60 \%$ de los encuestados consideran que éstas no se han llenado satisfactoriamente. Los aspectos más cuestionados de la calidad de la docencia fueron cumplimiento y metodología, mientras el contenido fue calificado como bueno. Con respecto a los recursos de infraestructura, el $57 \%$ opinan que las aulas son regulares o malas. El $54.4 \%$ opina que la disponibilidad de medios audiovisuales es adecuada. El 57\% considera que los sitios y elementos de consulta son regulares o malos.

Con relación al contenido práctico, el 84.3\% lo calificó como bueno o excelente. Solamente un 15\% denotó insatisfacción con la parte práctica de su programa. Las expectativas se consideran cumplidas en un $82 \%$ de los encuestados.

No existieron diferencias significativas en cuanto al nivel de residencia y la evaluación del programa.

Al preguntar por el porcentaje del tiempo en que cada residente practica labores asistenciales bajo supervisión directa de un docente, el 48\% refirió que no recibe supervisión docente en más del $50 \%$ de su tiempo de practica. Un $11 \%$ refirió tener supervisión docente en menos del 20\% del tiempo de su actividad asistencial.

El $87 \%$ de los residentes opinan que los hospitales no valoran su actividad asistencial aduciendo varios factores:

1. Carencia de estímulos como contraprestación o remuneración económica.

2. Ausencia de bienestar estudiantil y de áreas de descanso.

3. Sobrecarga asistencial para el residente quien aporta su mano de obra gratuita y siempre disponible, para suplir las deficiencias de personal de las instituciones.

$\mathrm{Al}$ indagar por derechos básicos de los residentes contemplados por la ley, como son: parqueadero, alimentación, ropa de trabajo, zonas de descanso, acceso a información médica, seguros de responsabilidad civil y riesgos profesionales y seguridad social, los residentes respondieron:

- $\quad 47 \%$ de los encuestados deben pagar su parqueadero.

- 20\% no tienen paga la alimentación.

- $20 \%$ no se les suministra ropa de trabajo.

- $31 \%$ refieren no tener acceso a zonas de descanso o bienestar:

- $54 \%$ no tienen acceso adecuado a información médica.

- $\quad 90 \%$ de los encuestados no tienen conocimiento de la existencia, o no están cubiertos por seguros de responsabilidad civil; y en un $88 \%$ por algún seguro de riesgos profesionales.

- $69 \%$ de los residentes refirieron no recibir cobertura de salud.
Cuando se pregunta por la idoneidad del proceso de selección de nuevos residentes, el $47 \%$ de los encuestados considera que este proceso no garantiza la calidad personal y profesional de los aspirantes. Entre las recomendaciones más frecuentemente referida por los inconformes están:

1. La carencia parcial o total de una valoración psicológica de los aspirantes.

2. Evaluar con mayor profundidad la hoja de vida.

3. La constante presencia del tráfico de influencias, recomendaciones y favores políticos.

4. Reducir el valor de la entrevista por su subjetividad y posibilidad de favorecimientos.

5. Garantizar la idoneidad y la capacitación del personal que hace la selección y que se respete sus decisiones y recomendaciones.

6. Ausencia de supervisión del proceso de selección por personal ajeno a la Universidad.

7. Fallas en la honestidad y transparencia en el proceso.

Al interrogar sobre los elementos que influyen en el ingreso de un individuo a su programa, el factor más importante fue el examen de conocimientos, seguido de las recomendaciones (léase palanca), en tercer lugar la entrevista, en cuarto lugar la hoja de vida y en último lugar la Universidad del pregrado. El $82.2 \%$ consideran que las "recomendaciones" influyen y hasta un 20\% consideran que es el elemento más importante. El 78\% consideraron que el sexo no tiene ningún valor como elemento para acceder a un programa.

Cuando se solicita a los encuestados expresar como se sienten respecto a su programa de residencia, respondieron así:

- Satisfechos: $30 \%$.

- Conformes: $43 \%$.

- Resignados: $26 \%$

- Frustrados: 1\%.

\section{Perfil del residente}

Encuestados acerca de sus actividades extracurriculares, el $38 \%$ le dedica tiempo a la lectura de temas no médicos (en los cuales primaron obras de García Márquez y bestsellers, temas "light"), un 43\% practican deporte y un $21 \%$ trabajan en su tiempo libre. Globalmente, un 84\% admitió tener tiempo libre de sus actividades académicoasistenciales.

Acerca de su intensidad asistencial horaria, el $84 \%$ de los residentes refirieron desempeñar jornadas de hasta 36 (treinta y seis) horas continuas en vigilia.

Con relación al manejo de otras lenguas, el Inglés fue el segundo idioma para los encuestados. Sólo 35\% refirió tener manejo completo del idioma (hablar, leer y escribir) mientras un $95 \%$ consideró tener conocimientos suficientes para su lectura de temas médicos. Aun así, la gran mayoría prefirió textos médicos en español como principal fuente de consulta. Menos del 5\% refirieron dominio de otras lenguas como el alemán, francés, hebreo, portugués e italiano. 
En cuanto al manejo de computadores, un $47 \%$ refirió dominio adecuado de procesadores de palabras, un $38 \%$ de graficadores de diapositivas, sólo un $21 \%$ conoce Internet y únicamente un 14\% maneja programas estadísticos.

$\mathrm{Al}$ encuestar sobre asistencia a eventos de educación continuada o actualización, menos del 50\% afirmó haber asistido en el último año a cualquiera de estos eventos. Sólo el $42 \%$ refirió haber asistido alguna vez a un congreso nacional de la especialidad.

$\mathrm{Al}$ indagar por publicaciones científicas en revistas locales, nacionales o internacionales, el 84\% manifestó nunca haber publicado un trabajo; aun así el 77\% manifiesta estar involucrados en trabajos de investigación clínica.

Al encuestar por manifestaciones psicosomáticas atribuibles a sus obligaciones académico-asistenciales, el $80 \%$ aceptó haber sufrido algún tipo de alteraciones: las manifestaciones gastrointestinales fueron las más frecuentes $(50 \%)$ con cuadros de gastritis, úlcera, reflujo y hemorroides. Posteriormente y en orden de frecuencia se refirieron: trastornos del sueño (39\%), pérdida de peso $(26 \%)$, disfunción sexual (9\%), alteraciones cardiovasculares (5\%) y cambios cónductuales como agresividad ( $5 \%$ ).

Al solicitar describir en pocas palabras los rasgos de personalidad del ginecobstetra como especialista los términos mas referidos en orden de frecuencia fueron: "buena gente", trabajador, responsable, paciente, obsesivo, estresado, amable, resignado y algunos lo consideraron como un "seductor" (! ?).

\section{Aspecto económico}

Al encuestar sobre pago de la matrícula, el $96 \%$ de los residentes pagan matrícula; de éstos el $86 \%$ cancelan valores entre 1 millón y 3 millones de pesos por semestre. El $64 \%$ de los residentes confiesan tener un apoyo familiar para cancelar sus estudios, el $37 \%$ se mantienen con ahorros y el $31 \%$ acuden al crédito educativo. Un $16.5 \%$ trabajan para financiar sus estudios.

El $80 \%$ de los residentes refieren que su programa no les permite trabajar, pero a pesar de esto un $26 \%$ refieren que lo hacen para cancelar sus becas y mantener a su familia. El área más frecuente de trabajo es la misma especialidad (80\%). Un 38\% refieren tener personas a su cargo.

\section{Percepción de la situación médica}

El $65 \%$ de los encuestados califica su conocimiento de la ley 100 con un puntaje de 3 o menos (1-5). A pesar de esto, la gran mayoría de encuestados la responsabilizan de ser la causa de la mala situación médica actual. En un segundo lugar consideran al gremio médico como una causa determinante en la situación médica actual.

El 82.6\% volvería a estudiar medicina. E1 98\% consideraría especializarse nuevamente. El 98\% volvería a escoger ginecobstetricia. Sólo el $73 \%$ escogería el mismo programa (el 27\% cambiaría el programa!!).

El $77.2 \%$ considera que la subespecilización es importante en nuestro medio. La subespecialidad más apetecida fue la medicina materno-fetal seguida de la infertilidad/ laparoscopia. En tercer lugar, la opción de no subespecializarse. En orden de frecuencia siguieron oncología, endocrinología y por último gineco-urología.

En cuanto a expectativas de remuneración una vez termine su residencia, la gran mayoría afirmó que consideraba unos ingresos de 2-4 millones como lo esperado. Un 12\% considera ganará menos de 2 millones y un 3\% más de seis.

Respecto a los intereses laborales una vez termine su residencia, la gran mayoría manifestó que estarían interesados en: (en orden de frecuencia): iniciar una subespecialidad, práctica privada, práctica hospitalaria y en último lugar ser docente.

La gran mayoría desearían trabajar en área urbana (68\%), mientras el restante $27 \%$ en provincia y el $5 \%$ en el exterior.

El $90 \%$ de los encuestados manifestaron nunca haber estado involucrados en demandas por responsabilidad médico legal.

El 71\% están de acuerdo con el aborto terapéutico bajo indicación médica. Un 7\% lo consideran como una opción viable de planificación familiar (!) y un $22 \%$ no están de acuerdo con ningún tipo de aborto.

Finalmente, al solicitar a los encuestados cómo calificarían el programa de residencia, el $44 \%$ le asigno un valor de $4 / 5$ mientras el $37 \%$ un valor de $3 / 5$. Sólo un $8 \%$ reprobaron su programa.

\section{Análisis}

\section{Identificación y datos generales}

Se observa que aún hoy en día el programa de ginecobstetricia es realizado en su gran mayoría por hombres $(77.5 \%)$. Un alto porcentaje son casados $(35.5 \%)$ lo cual supone mayores responsabilidades económicas que las del residente soltero. Llama la atención el encontrar casi un $35 \%$ de las encuestas sin datos de identificación. Lo cual si bien era una opción, puede también sugerir que el residente no quiere ser identificado para no herir susceptibilidades, o en el peor de los casos por que sus opiniones pueden derivar en retaliaciones.

\section{Del programa de residencia}

De los residentes que ingresan al programa de GinecoObstetricia, el $70 \%$ se presentaron para admisión más de dos veces. Un promedio alto que puede reflejar dos cosas: la dura competencia dada por un número alto de aspirantes y/o que el médico general no se presenta adecuadamente preparado a su intento inicial.

La mayoría de los aspirantes (52\%) aplican a un programa sin conocer adecuadamente su contenido; la prioridad parece ubicarse rápidamente en la especialización. Esto sugiere que el contenido no es lo primordial en el momento de escoger y aplicar a un determinado programa.

Llama la atención que un alto porcentaje de los residentes $(61 \%)$ que cursan actualmente programas de resi- 
dencia de 4 años, refieren que no encuentran ninguna ventaja que su programa sea de mayor duración. Las razones que aducen son: (1) incumplimiento del programa académico, (2) carencia de infraestructura física y de recurso docente para ejecutar este tipo de propuesta. (3) La mayoría concuerdan en que en realidad se lleva a cabo un programa de 3 años extendido a 4 años, (4) con perjuicios como aumento en la carga asistencial y una (5) demora injustificada en el inicio de su etapa productiva.

Las expectativas académicas no se han cumplido para la mayoría de los encuestados. Consideran que hay deficiencias en el cumplimiento de éstas por carencias en el recurso humano y en la metodología docente. Así mismo, refieren inconformidad por la deficiencia en recursos físicos como salones de clase y material de consulta. En resumen, la mayoría de encuestados opinan que las diferentes instituciones educativas y hospitales que tienen programas de residencia en Ginecología y Obstetricia, no se encuentran organizadas ni estructuradas para cumplir a cabalidad su objetivo académico-docente. Es relevante que la mayoría coincide en que la práctica asistencial se cumple. es suficiente y de buena calidad.

Es preocupante que los residentes coinciden en manifestar que la enseñanza basada en el concepto de "supervisión docente" durante la práctica asistencial, es insuficiente y no se cumple.

Es generalizada entre los residentes la opinión de que su actividad asistencial no es valorada por parte de los hospitales. Las carencias en lo que se refiere a bienestar estudiantil como zonas de descanso, disponibilidad de parqueo, alimentación y ropa de trabajo, son una constante. Así mismo aquejan sobrecarga de labores asistenciales sin compromiso de la institución por contraprestar su trabajo. Existe una reglamentación previa a este respecto contemplada en el decreto 190 del 25 de enero de 1996, que en su capítulo III titulado Convenios y Programas, presenta en su Parágrafo 2 la siguiente reglamentación:

"El convenio deberá incluir la reglamentación respectiva para que las instituciones involucradas en la relación docente asistencial respondan por el instrumental, equipo médico-quirúrgico del cual hagan uso y su mantenimiento durante el tiempo que dure el convenio. Igualmente deberá estipularse la forma de financiación de la alimentación, ropa de trabajo, uso de parqueaderos, áreas de descanso y otros".

Finalmente otro hallazgo preocupante que nos lleva a cuestionarnos sobre la calidad actual de los programas vigentes es el hecho de que solamente el $30 \%$ de los residentes refirieron estar satisfechos con su programa; ocho individuos $(1 \%)$ inclusive llegaron a manifestarse como frustrados por su programa.

\section{Perfil del residente}

Se logró determinar que el $84 \%$ de los residentes desempeñan jornadas hasta de 36 horas continuas, que a nuestro concepto disminuyen su rendimiento, limitan su capaci- dad de razonar y atentan contra el juicio clínico. Hasta qué punto es conveniente que se mantengan éstas jornadas en la residencia más aún teniendo en cuenta que la legislación laboral expresamente las prohibe.

Aunque un porcentaje alto de residentes manifiesta tener algún grado de dominio del idioma inglés, sólo el 35\% tiene un manejo completo que incluye el leerlo, escribirlo y hablarlo. Muy posiblemente el conocimiento del inglés es tan insuficiente que por eso se prefieren los textos en español como principal fuente de consulta.

En general, el manejo de computadores y software accesorio es muy precario.

En este punto vemos otra deficiencia de los programas de residencia, pues no se capacita al residente en el dominio de esta tecnología que a corto o mediano plazo indudablemente será parte esencial de su desempeño como ser humano y profesional.

Otro aspecto que no deja de sorprender, es el hecho de que los residentes no asisten masivamente a las actividades académicas de la especialidad; ya sea por que no hay motivación o por que su tiempo libre lo deben emplear trabajando o descansando. También se debe anotar que generalmente los hospitales no facilitan la asistencia del residente a estas actividades. Aquí es cuando parece olvidarse que la esencia en la formación del residente son las actividades académicas, por sus estatus de estudiante y de individuo en formación.

A pesar de que la mayoría de residentes $(77 \%)$ se hayan involucrados en trabajos de investigación clínica, un número limitado (15\%) han publicado en revistas de la especialidad. De esto se puede asumir que existen deficiencias en la calidad científica de los trabajos y/o no existe interés del residente en publicar. Esto sugiere que probablemente los trabajos son solamente un requisito necesario para avanzar un año en la residencia u obtener un diploma. Hasta qué punto las universidades y hospitales se han preocupado en capacitar desde el punto de vista investigativo a los residentes, motivándolos a producir trabajos que trasciendan teniendo verdadero valor académico, utilidad clínica y beneficio para su hospital y comunidad.

El residente durante su entrenamiento se ve afectado por el estrés propio de la actividad laboral (responsabilidades académicas, asistenciales y económicas), lo cual en últimas nos lleva a que más del $80 \%$ de ellos hayan presentado alguna manifestación psicosomática que indudablemente afecta su desempeño académico-asistencial. Nos preguntamos, ¿no es éste un síntoma inequívoco de la sobrecarga en responsabilidades a las que se está sometiendo al estudiante de postgrado? ¿O por el contrario, aprender a manejar estos niveles de estrés debe hacer parte de su formación como profesional?

Cuando se solicitó definir el perfil del Gineco-obstetra, la mayoría lo describió como una persona amplia, tolerante y trabajadora, con una admirable adaptabilidad a las condiciones de trabajo. Ocasionalmente se refirieron algunas manifestaciones neuróticas y trastornos emocionales. 


\section{Aspecto económico}

E1 96\% de los residentes deben pagar una matrícula que oscila entre un millón y tres millones de pesos por período académico de seis meses. Para poder cumplir con estos elevados requerimientos económicos y garantizar su formación profesional, los residentes acuden principalmente al apoyo familiar, a sus ahorros, al crédito educativo y algunos trabajan.

A pesar que al $80 \%$ de los residentes su programa no les permite trabajar, un 26\% lo hace para cumplir sus obligaciones económicas; de éstos un 17\% lo hacen primordialmente para pagar sus estudios. No debemos olvidar que el $38 \%$ de los encuestados tienen otras personas que dependen económicamente de ellos. Afortunadamente, la gran mayoría de las personas que laboran lo hacen en el área de la especialidad.

\section{Percepción de la situación médica}

Una gran parte de los participantes en este estudio manifiestan un desconocimiento casi total respecto a la Ley de Seguridad Social; aún así la responsabilizan de la situación actual de nuestra profesión. Al mismo tiempo consideran que el mismo médico es responsable de alguna manera de la situación actual que vive la medicina.

A pesar de lo anterior parece existir una gran vocación por la medicina entre los encuestados; en un alto porcentaje $(83 \%)$ volverían a estudiarla. Casi todos $(98 \%)$ volverían a escoger la Gineco-obstetricia como especialidad. Como dato. importante, un $27 \%$ no volverían a escoger su mismo programa académico.

Llama la atención que se considera importante la subespecialización, pero no es una prioridad, ya que a estas alturas muy pocos están dispuestos a seguir estudiando formalmente, en vez de empezar a producir. Las subespecialidades más buscadas por los residentes son la Medicina Maternofetal y la Infertilidad.

Las expectativas de ingresos están muy ajustadas a la realidad de la especialidad en el medio y en general de la medicina. Persiste el deseo de trabajar en la capital más que en la provincia.

En la parte final de la encuesta, al solicitar una evaluación del programa de residencia, llama la atención de que a pesar de haber criticado en muchos aspectos los respectivos programas, sólo un $8 \%$ de los encuestados los reprobaron. Parece existir un curioso sentimiento ambivalente de: (1) tidelidad y sentido de pertenencia con su universidad con el de: (2) inconformismo con el programa, que muy posiblemente vicia la evaluación final. Pareciera que el residente es consciente de las deficiencias de su postgrado pero a la vez quiere a su Universidad y le duele calificarla mal.

\section{Reflexiones}

Existió una buena acogida de esta encuesta entre los residentes ya que proporcionó un escenario apropiado para manifestar sus inconformidades. Se creó la expectativa que esta encuesta pudiera abrir la posibilidad a cambios que beneficien los programas de postgrado y por ende a los residentes. Un 36\% de los evaluados optó por el anonimato al diligenciar la encuesta; nos preocupa que esto sea el reflejo de un temor del residente por expresar abiertamente sus opiniones.

Hoy en día los residentes de gineco-obstetricia en Colombia son personas que han decidido enfrentar muchas dificultades por ingresar a su programa. En su mayoría no se arrepienten de estar estudiando gineco-obstetricia, pero se plantean serias dudas acerca de la calidad académica de sus programas. Consideran que la rica actividad asistencial puede equilibrar de alguna forma el aporte académico deficiente. Especulamos que la residencia se está convirtiendo en un aprendizaje en base en la práctica primordialmente, lo que nos recuerda el concepto antiguo de formación empírica, antes de que las especializaciones fueran tomadas por instituciones educativas. Es inobjetable el efecto que ha tenido la situación actual de la medicina en Colombia sobre la formación académica.

En general los residentes son considerados mano de obra de bajo costo para cubrir las crecientes obligaciones asistenciales de un hospital que asume su formación bajo un convenio docente con una entidad educativa. Por su carácter de estudiante no se les reconoce condición laboral alguna, ni las prebendas previstas por la ley como Seguridad Social, cobertura contra Riesgos Profesionales y Responsabilidad Civil. Así mismo tampoco se han respetado los beneficios pactados en la Ley de Convenios y Programas.

El desinterés del residente por manifestarse o siquiera discutir sobre estos tópicos posiblemente obedece a factores como: (1) su status temporal como estudiante, (2) los inconvenientes por los que pasó para acceder al programa de residencia, (3) el riesgo de indisponerse con sus superiores y (4) los costos propios de las matrículas; estos factores promueven su apatía y más pareciera estar dispuesto a tolerar en silencio esta situación por tres a cuatro años, antes de buscar un cambio que beneficie a los que le siguen. Además las características de su personalidad lo convierten en el elemento laboral ideal para los hospitales de nuestro nuevos sistemas de seguridad social donde prima la productividad.

Dentro del proceso de formación.del especialista existe una secuencia de eventos que podrían antojarse contradictorios o inclusive irónicos. Para el médico general es una prioridad ingresar al programa de residencia, quizás pretendiendo elevar su estatus científico y médico; o posiblemente considerando mejorar sus expectativas económicas. Se presenta sistemáticamente a diversos programas sin tener como prioridad su contenido académico, pero sí quizás muy pendiente de la posibilidad de contar con "ayudas" extra-académicas. Una vez como residente, asume un alto desgaste: se somete a una carga académico-asistencial muy exigente, compromete su salud, posterga su productividad, enfrenta el factor económico y riesgos profesionales, y en términos globales asume su rol de estudiante-trabajador; sin llegar a beneficiarse en la mayoría de las veces de los 
derechos que estas dos condiciones le deberían otorgar. De antemano el residente es consciente que será un profesional mal remunerado y que tendrá que competir en un medio hostil; aún así no se arrepiente de su decisión y aún más, considera la subespecialización como una posibilidad de su desempeño profesional. Posiblemente esta decisión obedece a un deseo de aumentar aún más sus conocimientos para ser un individuo más competitivo o simplemente por propia satisfacción profesional y personal.

Lamentablemente en el escenario médico que ha planteado la ley de seguridad social, bajo los esquemas actuales de atención médica y de comercialización de servicios profesionales, la idoneidad del profesional y su currículum de postgrado no son considerados como prioritarios en su desempeño profesional. Entonces nos cuestionamos si al momento de enfrentar este tortuoso proceso de formación, el estudiante ha hecho una decisión laboral inteligente.

Siendo el programa de residencia un producto de gran demanda y que por su naturaleza médica misma debiera tener los más altos estándares de calidad, es preocupante el que no exista hoy día un adecuado control sobre el contenido académico y cumplimiento de éste. Es de suma importancia crear mecanismos de autoevaluación dentro de los programas. Las sociedades científicas deberían coordinar sus esfuerzos a exigir que perdure la excelencia médica, ética y profesional; aún en el oscuro panorama de la práctica médica actual, en el que las mismas deben verse más comprometidas.

Esta encuesta cumplió con sus objetivos de desnudar las deficiencias y las fortalezas de los programas de residencia, sembrando inquietudes entre residentes y docentes inconformes. Los resultados observados en esta encuesta deben motivar a que las Universidades y sus postgrados se autoevalúen y se planteen cambios de fondo para hacerse más eficientes y acordes a la realidad de la medicina hoy en día. Tenemos claro que estas evaluaciones se deben poner en marcha antes de considerar la apertura de nuevos programas de residencia. 